\title{
Enhanced Eye Gaze Direction Classification Using a Combination of Face Detection, CHT and SVM
}

\author{
Amer Al-Rahayfeh and Miad Faezipour \\ Department of Computer Science and Engineering \\ University of Bridgeport, Bridgeport, CT
}

\begin{abstract}
Automatic estimation of eye gaze direction is an interesting research area in the field of computer vision that is growing rapidly with its wide range of potential applications. However, it is still a very challenging task to implement a robust eye gaze classification system. This paper proposes a robust eye detection system that uses face detection for finding the eyes region. The Circular Hough Transform (CHT) is used for locating the center of the iris. The parameters of the Circular Hough Transform are dynamically calculated based on the detected face information. A new method for eye gaze direction classification using Support Vector Machine (SVM) is introduced and combined with Circular Hough Transform to complete the task required. The experiments were performed on a database containing 4000 images of 40 subjects from different ages and genders. The algorithm achieved a classification accuracy of up to $92.1 \%$.
\end{abstract}

Motivation - Eye gaze classification systems aim to locate the eye in the image and then use the obtained information about the eye region and the head pose to estimate the eye gaze direction to be used in in various applications, such as Human Computer Interaction (HCl), virtual reality, driver assistance systems and assistance for people with disabilities.

System Overview - Viola-Jones is used as a first step in the system for face detection. Then, Circular Hough Transform is used to locate the iris. The detected face height can be used to calculate the minimum and maximum values of the radius used by $\mathrm{CHT}$ for iris detection. In addition, the eye region can be defined from the detected face area. This minimizes the number of detected iris candidates. The detected iris radius value is used in the preprocessing stage to extract a sub-image from the face image after it is converted to HSI scale.

Two regions are defined in the extracted sub-image: right and left regions. Each of these regions has a width and height equal to the iris diameter. The average of the $\mathrm{H}$ component from HSI scale for each region, the left and the right, can be used as a good feature for eye direction classification.

To determine whether the gaze direction is left or right, SVM classifier is used where the feature vector is the averages of the hue value of the left and right regions of the sub-image. The SVM was trained using a training dataset consisting of images captured for 40 subjects from different ages and genders.
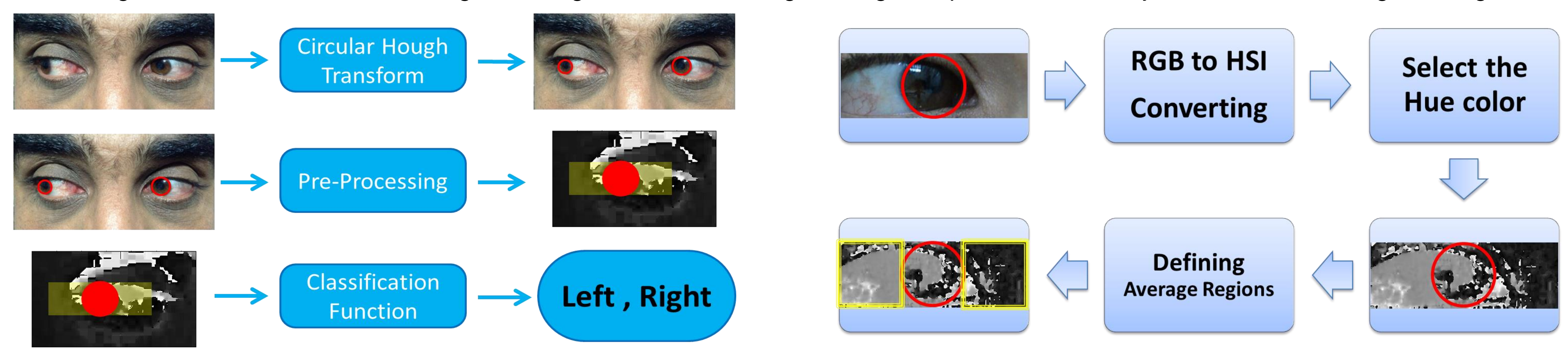

Viola-Jones Face Detection = It included three main concepts which led to successful real-time face detection. These three concepts are:

- The Integral Image: an intermediate image presentation which allows fast evaluation of the features.

- AdaBoost: it is used to select a small number of the many computed features.

- The Attentional Cascade: it is used to cascade classifiers to reduce computation time and improve the accuracy of detection.

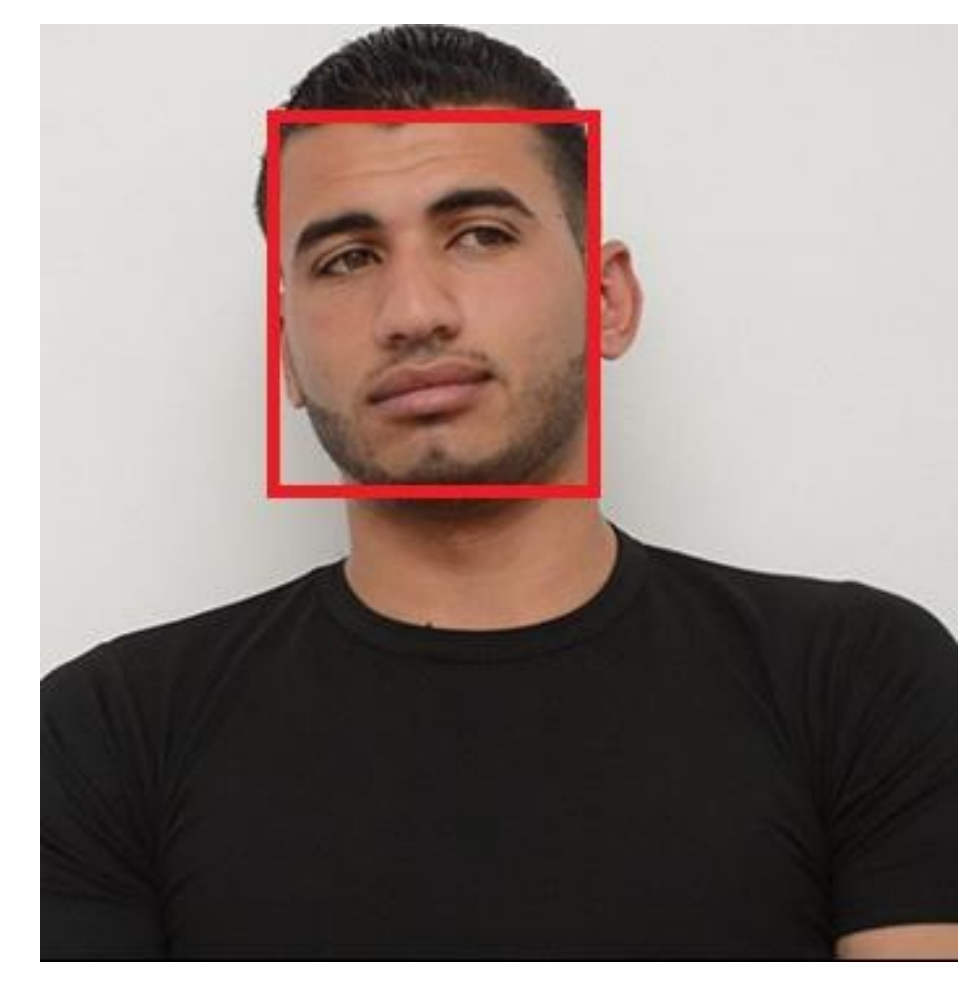

Circular Hough Transform - $(\mathrm{CHT})$ is a modified version of the Hough Transform that recognizes circular patterns in the processed image. These patterns may be a complete circle or a circular curve. It transforms the sets of feature points in the image into sets of accumulated votes in parameter space. For each feature point, votes are accumulated in an array which spans all parameter combinations. The highest number of votes indicates that there is a shape.

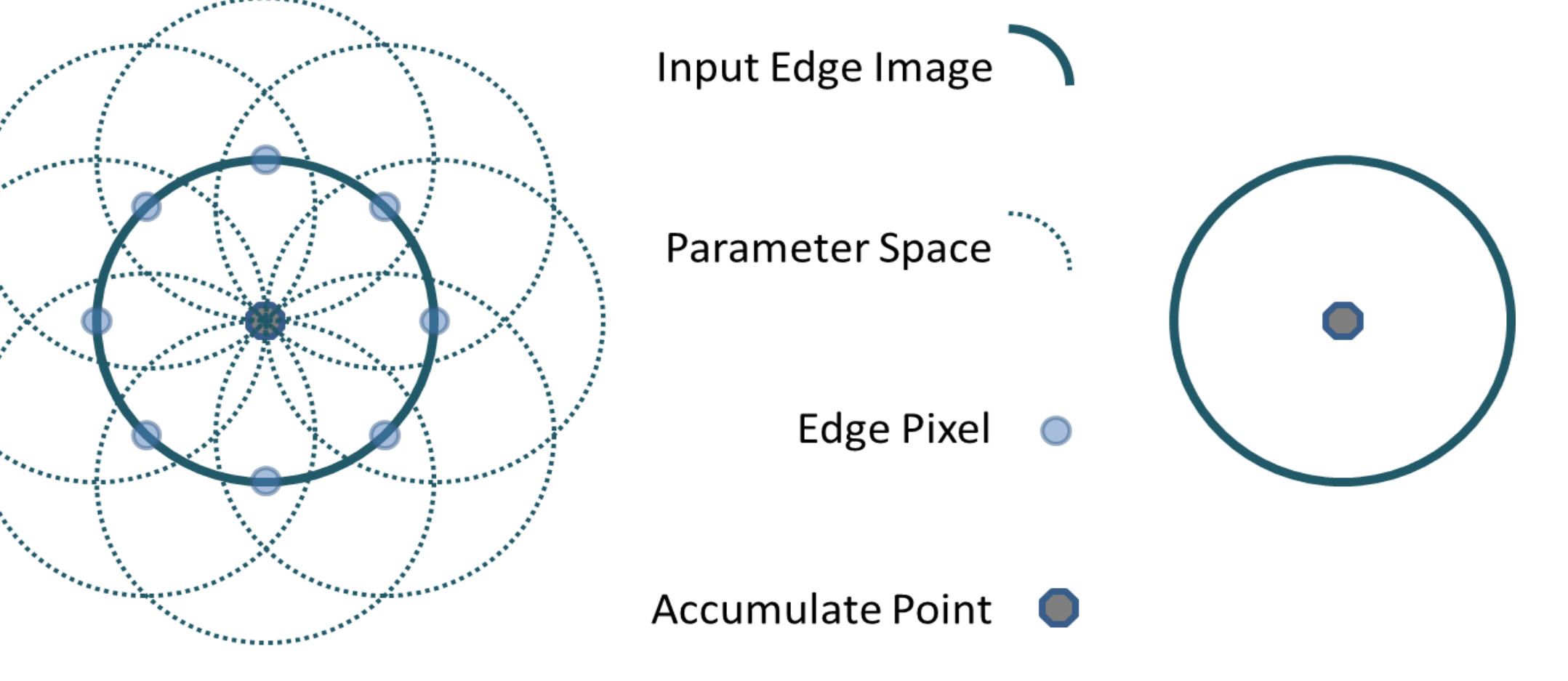

Support Vector Machine- SVMs are supervised learning models based on some learning algorithms in which data is analyzed and used in classification. The input data is mapped into an $\mathrm{N}$ dimensional space using a kernel function. The data points existing on the margin are called the support vectors. These support vectors are used by SVM to define the optimal separating hyper-plane between the classes of input data which leaves the maximum margin from both classes.

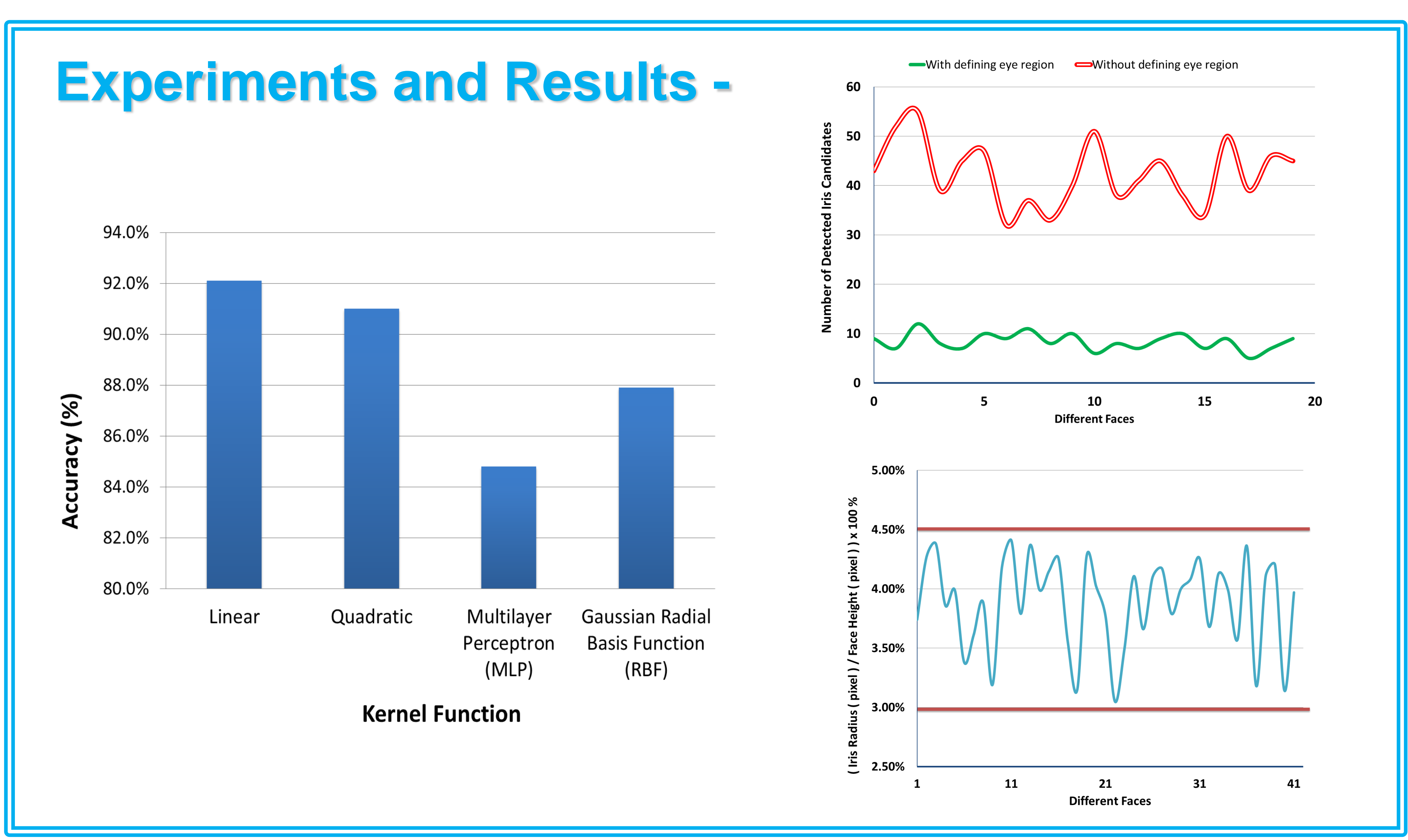

